\title{
The Pore Collapse "Hot-Spots" Model Coupled with Brittle Damage for Solid Explosives
}

\author{
L. R. Cheng, ${ }^{1}$ R. Chen, ${ }^{2}$ H. J. Shi, ${ }^{1}$ and F. Y. $\mathrm{Lu}^{2}$ \\ ${ }^{1}$ Department of Engineering Mechanics, School of Aerospace, AML, Tsinghua University, Beijing 100084, China \\ ${ }^{2}$ College of Science, National University of Defense Technology, Changsha 410073, China \\ Correspondence should be addressed to L. R. Cheng; ruirui20041127@sina.com
}

Received 15 May 2014; Revised 9 September 2014; Accepted 10 September 2014; Published 4 December 2014

Academic Editor: Vadim V. Silberschmidt

Copyright (C) 2014 L. R. Cheng et al. This is an open access article distributed under the Creative Commons Attribution License, which permits unrestricted use, distribution, and reproduction in any medium, provided the original work is properly cited.

\begin{abstract}
This paper is devoted to the building of a numerical pore collapse model with "hot-spots" formation for the impacted damage explosives. According to damage mechanical evolution of brittle material, the one-dimensional elastic-viscoplastic collapse model was improved to incorporate the impact damage during the dynamic collapse of pores. The damage of explosives was studied using the statistical crack mechanics (SCRAM). The effects of the heat conduction and the chemical reaction were taken into account in the formation of "hot-spots." To verify the improved model, numerical simulations were carried out for different pressure states and used to model a multiple-impact experiment. The results show that repeated weak impacts can lead to the collapse of pores and the "hot-spots" may occur due to the accumulation of internal defects accompanied by the softening of explosives.
\end{abstract}

\section{Introduction}

Numerous studies show that the impact of energetic materials can lead to delayed detonation, which is commonly referred to as XDT events. XDT usually occurs under weak and moderate waves in pressed solid explosives. Compared with the shock initiation to detonation (SDT), the threshold pressure of XDT is significantly lower and the detonation time is considerably longer. A lot of researches which focused on XDT phenomenon have been carried out in order to evaluate and improve the safety and invulnerability of weapons [14]. Although the specific mechanism of XDT is not clear, many testing results show that the pressed solid explosives probably become more sensitive due to impact damage and that the subsequent secondary compression wave can cause detonation to happen easily. Recently, some studies on XDT are focusing on the formation of "hot-spots" caused by impact damage of explosives [1-3].

Until now many "hot-spots" mechanisms, such as pore collapse, friction, and shear bands, have been investigated [4-8]. Among them, the pore collapse mechanism is widely researched because this mechanism can produce much more energy than the others during the impact initiation [4]. It is assumed that "hot-spots" are formed as a result of the elasticviscoplastic collapse of the pores in the solid explosives under shock loading. Many improvements based on the elasticviscoplastic pore collapse model have been performed in order to consider different physical mechanisms. The initial porosity effects on detonation can be shown by the 1D hollow micromechanical pore collapse model, which was developed and applied to reaction rate function by Kim [9]. Considering that the viscoplastic work was a more efficient heating mechanism for "hot-spots" in the vicinity of the collapsed pore, Whitworth $[10,11]$ divided the process of pore collapse into three possible phases. Furthermore, Zhang et al. [12] considered the melting effects during the collapse of the pores; Wen et al. [13] developed the model of doublelayered hollow sphere in order to consider the viscous effects of binder materials on the formation of "hot-spots."

The present elastic-viscoplastic collapse models are mostly based on the ductile mechanical property based on Carroll-Holt's model [14]. On the contrary, many experimental results show that explosives, such as PBXs, have typical brittle mechanical properties under impact loading [15-17]. The initial defects or damage in the explosives is inevitable in the process of production or usage. The growth and spread 
of these defects and damage play an important role in the formation of "hot-spots" [18]. Wu and Huang [19] developed a micromechanics model to describe "hot-spots" formation in the energetic crystal powders subjected to drop-weight impact. In the present study, the one-dimensional elasticviscoplastic collapse model was improved by considering the growth of damage in the pressed explosives.

This paper proposes a pore collapse model with "hotspots" formation for the impacted damage explosives. Firstly, the effect of damage of explosives is added into the elasticviscoplastic collapse model based on Whitworth model (Section 2). Secondly, the formation of "hot-spots" in two typical cases was investigated by the improved model. One case is that the explosive is loaded by a weak pressure for longer time; the other is that the explosive is loaded by multiple repeated weak pressures for shorter time (Section 3). Thirdly, we simulate the multiple repeated impact test by Gao et al. [20] to validate the improved model (Section 4). Finally, some discussions and remarks are given (Section 5).

\section{Improvement on Elastic-Viscoplastic Pore Collapse Model}

2.1. The Whitworth Model. The one-dimensional hollow spherical model was shown in Figure 1 according to the Whitworth model $[10,11]$, where $a_{0}$ and $b_{0}$ are the initial inner radius and external radius, respectively. The initial distention ratio $\varphi_{0}=b_{0}^{3} /\left(b_{0}^{3}-a_{0}^{3}\right)$ is the volume ratio of the total porous material compared to the solid material. The initial parameter of the hollow spherical model can be obtained by the average pore radius and initial overall porosity of the explosives. The time evolution of the pore radius is given as follows:

$$
-\rho\left(a \ddot{a}+1.5 \dot{a}^{2}\right)=P_{s}+P_{v}-P_{y}
$$

where $\rho$ is the density of the solid explosive, $a$ is the realtime inner radius of the pore, $P_{s}$ is the applied pressure, $P_{v}$ is the viscous stress, $P_{y}$ is the elastic-plastic stress, and $\dot{a}$ and $\ddot{a}$ denote a derivative with respect to time.

In the Whitworth model, the deformation of the pore can be divided into three phases.

(i) Elastic phase $\left(\varphi_{0} \geq \varphi \geq \varphi_{1}\right)$ :

$$
P_{\nu}=0, \quad P_{y}=\frac{4 G\left(\varphi_{0}-\varphi\right)}{3 \varphi(\varphi-1)}, \quad \varphi_{1}=\frac{2 G \varphi_{0}+Y}{2 G+Y} .
$$

(ii) Elastic-viscoplastic phase $\left(\varphi_{1} \geq \varphi \geq \varphi_{2}\right)$ :

$$
\begin{gathered}
P_{v}=12 \eta a^{2} \dot{a} \int_{a}^{d} \frac{1}{r^{4}} d r, \quad \varphi_{2}=\frac{2 G \varphi_{0}}{2 G+Y}, \\
P_{y}=\frac{2}{3} Y\left(1-\frac{2 G\left(\varphi_{0}-\varphi\right)}{Y \varphi}+\ln \left\{\frac{2 G\left(\varphi_{0}-\varphi\right)}{Y(\varphi-1)}\right\}\right),
\end{gathered}
$$

where $d$ corresponds to the time-dependent interface between elastic and viscoplastic flow, which is introduced as

$$
d=\sqrt[3]{\frac{2 G B}{Y}}, \quad B=\frac{\varphi_{0}^{3}\left(\varphi_{0}-\varphi\right)}{\varphi_{0}-1} .
$$

(iii) Viscoplastic phase $\left(\varphi_{2} \geq \varphi \geq 1\right)$ :

$$
P_{v}=12 \eta a^{2} \dot{a} \int_{a}^{b} \frac{1}{r^{4}} d r, \quad P_{y}=\frac{2}{3} Y \ln \left(\frac{\varphi}{\varphi-1}\right),
$$

where $G$ is the shear modulus, $Y$ is the yield strength, $\eta$ is viscosity, $b$ is the real-time external radius, $r$ is the radial position, $\varphi_{0}$ is the initial distention ratio, and $\varphi$ is the current distention ratio, $\varphi=b^{3} /\left(b^{3}-a^{3}\right)$.

The increase of the temperature at the radial position $r$ as a result of the mechanical deformation during pore radial motion is given as

$$
\rho C_{v} \dot{T}=12 \eta\left(\frac{u^{2}}{r^{2}}\right)+2 Y\left(\frac{|u|}{r}\right), \quad u=\frac{\dot{a} a^{2}}{r^{2}},
$$

where $T$ is the local temperature, $C_{v}$ is the specific heat capacity, and $u$ is the local velocity in the solid shell.

2.2. The Improved Model. As reported [4, 21, 22] by many researchers that there are many irregular defects or cracks in the pressed explosives (such as PBX9404 [21] and PBX9501 [22]), for simplicity, it is assumed that there are some defects or cracks in the shell in the improved model. The initial volume of pore is defined as same as that in the Whitworth model, which represents the average pore radius and overall porosity of the explosives. The pore collapse can form "hotspots." The property of the explosives in the shell can be affected by the cracks. The effect of cracks is constructed in this paper following the Visco-SCRAM model developed by Bennett et al. [22]. In addition, only the expansion of the cracks is considered while the initiation of crack is ignored. The expansion of these cracks can lead to some degradation of the mechanical property of the explosives, which would promote the collapse and the formation of "hot-spots." The heating effect is involved in the collapse process when the explosive is softened due to the expansion of cracks. In the paper, the heating effect of the pore collapse is considered as the main effect and the heating effect of the crack is ignored.

Based on the damage mechanics, it is known that the initial defects or cracks in the explosives will begin to spread when the stress intensity exceeds the fracture toughness. As shown in Figure 2, the microcracks near the internal radius of the pore will be developed firstly with orthogonal distribution, because the max stress intensity will appear in the internal radius of the pore under the external pressure. These cracks are 45 degrees to the radius of the hollow pore. The expansion of these cracks can lead to degradation of the mechanical property which would promote the collapse. In this paper, the material mechanical property was mainly concerned with the overall effect of all the cracks, rather than the expansion of single crack.

As noted above, in the Whitworth model, the state of the pressed pore was divided into three stages: elastic, elasticviscoplastic, and viscoplastic. In the elastic state, since the stress is too small to make the initial defects or cracks grow rapidly, the influence of cracks on material mechanic property can be ignored. In the viscoplastic state, the pore collapse is completed rapidly within a few microseconds and 


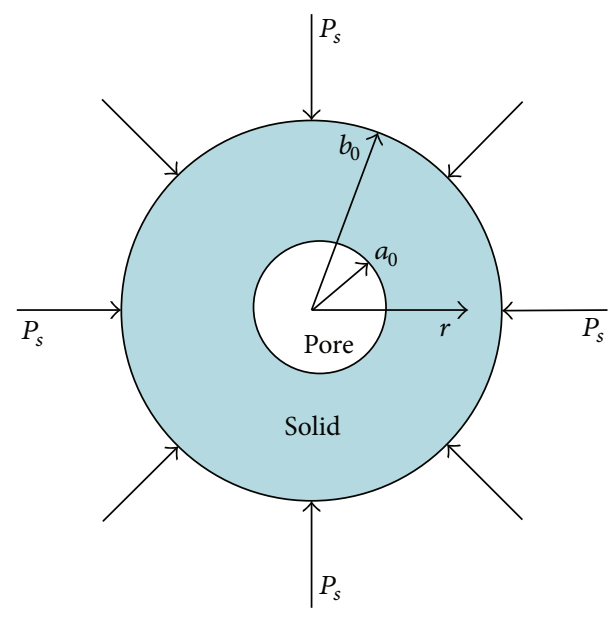

(a) The initial state

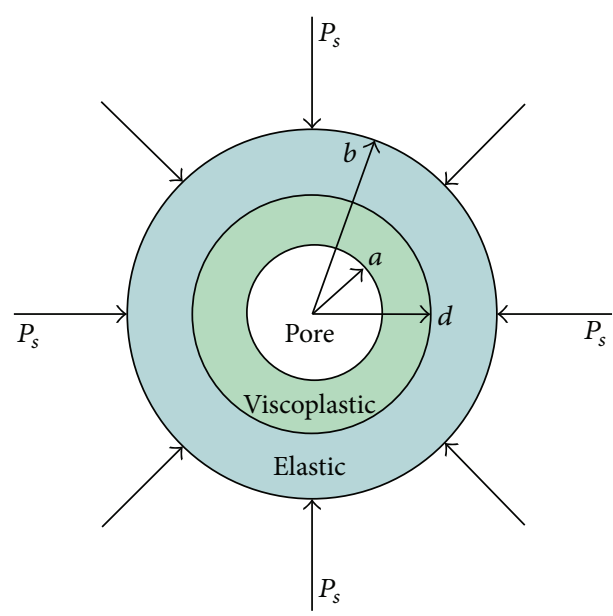

(b) The real-time state

FIGURE 1: One-dimensional hollow spherical model for "hot-spots" formation ( $d$ denotes the boundary between elastic and viscoplastic flow).
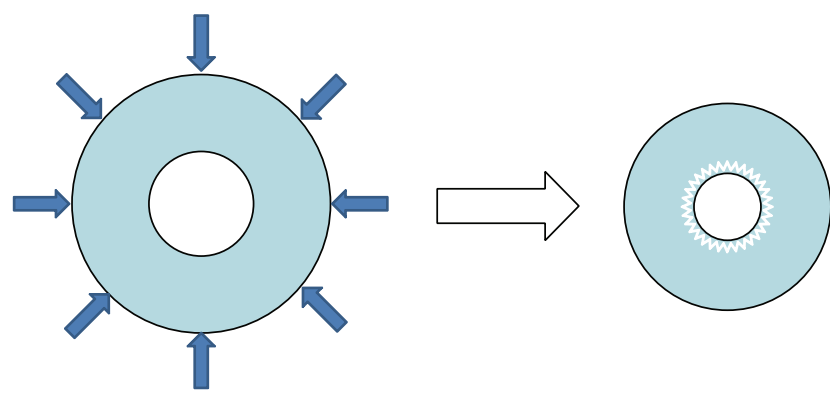

FIGURE 2: Hollow pore collapse coupled with damage extension under external pressure.

the initial cracks have no enough time to spread. Therefore, the expansion of cracks and mechanical property degradation are mainly concerned with the elastic-viscoplastic state in the improved model.

In this paper, the mechanical property degradation is described using the damage level $(D)$. In a micromechanical material model, the statistical crack mechanics (SCRAM) was developed by Dienes and his coworkers [22-26] to predict the dynamic mechanical deformation and fragmentation of brittle materials based on micromechanical statistical approach. Based on SCRAM, the Visco-SCRAM model was developed by Bennett et al. [22], which can be used to get the damage level $(D)$ [23]. It is assumed that the growth rate of cracks depends on the stress intensity according to ViscoSCRAM model:

$$
\begin{gathered}
\dot{c}=v_{\max }\left(\frac{K}{K_{1}}\right)^{m}, \quad K<K_{0} \sqrt{1+\frac{2}{m}}, \\
\dot{c}=v_{\max }\left(1-\left(\frac{K_{0}}{K}\right)^{2}\right), \quad K \geq K_{0} \sqrt{1+\frac{2}{m}},
\end{gathered}
$$

$$
\begin{aligned}
& K_{1}=K_{0} \sqrt{1+\frac{2}{m}}\left(1+\frac{2}{m}\right)^{1 / m}, \\
& K^{2}=\pi \cdot c \cdot \sigma_{\mathrm{eff}}^{2}=\frac{3}{2} \pi \cdot c \cdot S_{i j} S_{i j},
\end{aligned}
$$

where $c$ is average crack radius, $K_{0}$ is the fracture toughness of the explosives, $K$ is the stress intensity factor, $v_{\max }$ is the terminal speed of crack growth, $m$ is a material constant, $\sigma_{\text {eff }}$ is the Von-Mises stress, $S_{i j}$ is the deviatoric stress, and $S_{i j}$ denotes $S_{r}, S_{\theta}$, and $S_{\varphi}$. In Visco-SCRAM model, the damage level $(D)$ is expressed by the average crack radius $(c)$ :

$$
D=\frac{\left(c / c_{0}\right)^{3}}{1+\left(c / c_{0}\right)^{3}},
$$

where $c_{0}$ is the initial crack radius. Here, we assume that the initial crack radius is the same as the average pore radius and the value is $10 \mu \mathrm{m}$. The larger crack of initial radius causes the higher damage level $(D)$.

By including the damage level $(D)$ in (1) the improved model, the evolution of the pore radius is deduced as

$$
-\rho\left(a \ddot{a}+1.5 \dot{a}^{2}\right)=P_{s}+(1-D) P_{v}-(1-D) P_{y} .
$$

Furthermore, the temperature increase at the different position $(r)$ including heat conduction and chemical reaction is introduced as

$$
\begin{gathered}
\frac{\partial}{\partial r}\left(k \frac{\partial T}{\partial r}\right)+\rho \Delta H Z e^{E / R T}+Q=\rho C_{v} \frac{\partial T}{\partial t}, \\
Q=12 \eta\left(\frac{u^{2}}{r^{2}}\right)+2 Y\left(\frac{|u|}{r}\right),
\end{gathered}
$$

where $k$ is the solid conduction, $\Delta H$ is explosion energy, $Z$ is the frequency factor, $E$ is the Arrhenius activation energy, $R$ is the universal gas constant, and $t$ is the time. The first term in 


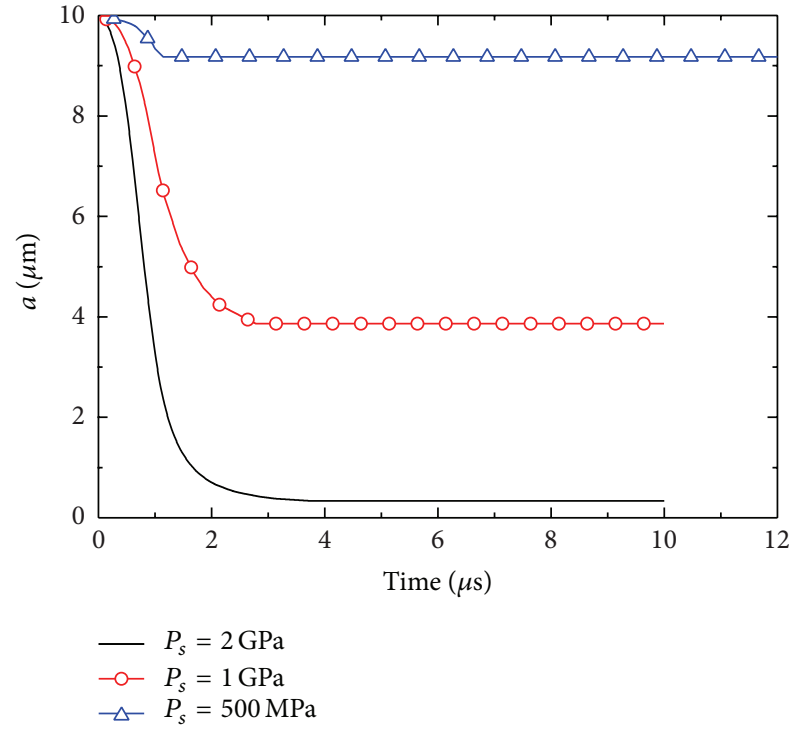

FIgURE 3: Time evolution of pore radius under three different external pressures with Whitworth model.

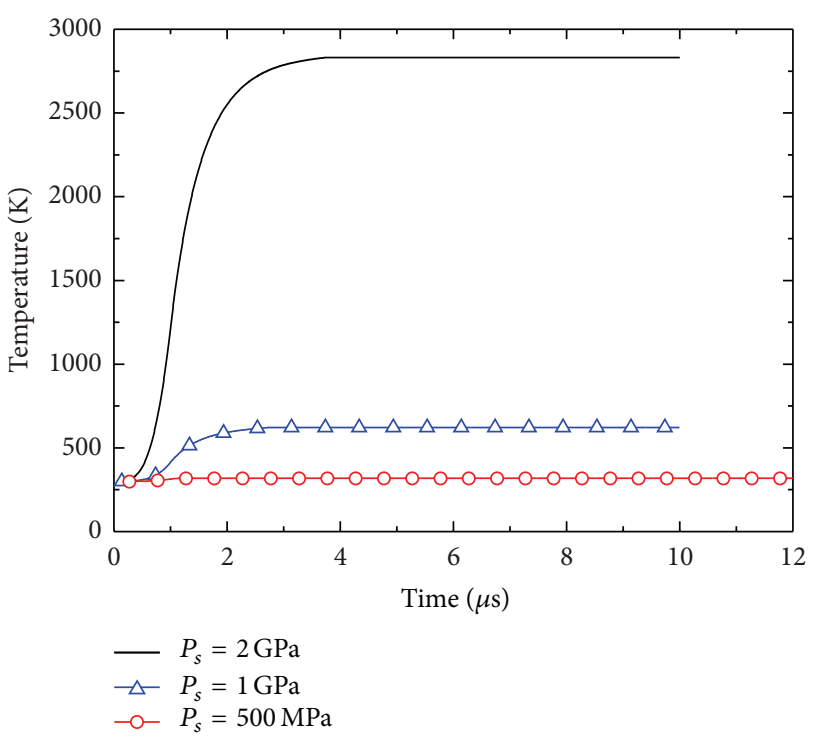

FIGURE 4: Time evolution of temperature at pore surface under three different external pressures with Whitworth model.

the left side of (10) denotes the heat outflow from "hot-spots," the second term represents the generated chemical heat, and the third term is the heat from the mechanical deformation. The right side of (10) detonates the heat stored in the area of "hot-spots."

\section{Application of the Improved Model}

In order to demonstrate the availability and validity of the improved model, the explosive PBX-9404 was selected to be simulated. Its main material parameters $[10,11]$ are listed in Table 1 . The pore radius $(a)$ and the maximum temperature
TABLE 1: Material parameters for PBX-9404.

\begin{tabular}{lc}
\hline Material parameter & value \\
\hline Initial density, $\rho\left(\mathrm{g} / \mathrm{cm}^{3}\right)$ & 1.84 \\
Yield strength, $Y(\mathrm{GPa})$ & 0.2 \\
Shear modulus, $G(\mathrm{GPa})$ & 4.54 \\
Viscosity, $\eta\left(\mathrm{GPa}^{*} \mu \mathrm{s}\right)$ & 0.1 \\
Initial temperature, $T_{0}(\mathrm{~K})$ & 300 \\
Specific heat, $C_{v}\left(\mathrm{GPa}^{*} \mathrm{~cm}^{3} / \mathrm{g} / \mathrm{K}\right)$ & $1.512 e-3$ \\
Frequency factor, $Z\left(\mu \mathrm{s}^{-1}\right)$ & $1.81 e+19$ \\
Activation energy, $E\left(\mathrm{GPa}^{*} \mathrm{~cm}^{3} / \mathrm{mole}\right)$ & 220.5 \\
Initial inner pore radius, $a_{0}(\mu \mathrm{m})$ & 10.0 \\
Initial external pore radius, $b_{0}(\mu \mathrm{m})$ & 46.416 \\
Initial crack radius, $c_{0}(\mu \mathrm{m})$ & 10.0 \\
Fracture toughness, $K_{0}\left(\mathrm{GPa}^{*} \sqrt{\mathrm{cm}}\right)$ & $1.0 e-2$ \\
Terminal speed for crack growth, $v_{\max }(\mathrm{cm} / \mu \mathrm{s})$ & $300 e-4$ \\
material constant, $m$ & 10 \\
\hline
\end{tabular}

$(T)$ in the hollow pore can be obtained according to different loading pressures, and these two parameters were different between the improved model and Whitworth model. Using the Whitworth model, the pore radius and the maximum temperature histories were predicted under the pressure of $P_{s}=500 \mathrm{MPa}, 1 \mathrm{GPa}, 2 \mathrm{GPa}$, as shown in Figures 3 and 4 . It can be seen that the pore radius can rapidly decline within two microseconds under the pressure of $P_{s}=1 \mathrm{GPa}$ and $2 \mathrm{GPa}$. The maximum temperature can increase to $650 \mathrm{~K}$ under $P_{s}=$ $1 \mathrm{GPa}$ and $2800 \mathrm{~K}$ under $P_{s}=2 \mathrm{GPa}$, respectively. While $P_{s}=500 \mathrm{MPa}$, the change of the pore radius is moderate and the maximum temperature cannot exceed $330 \mathrm{~K}$, which is lower than the melting temperature (usually $600 \mathrm{~K}$ ) of explosive. Therefore, it is concluded that "hot-spots" cannot occur under the weak pressure of $P_{s}=500 \mathrm{MPa}$ in the Whitworth model.

Using the improved model, the pore radius $(a)$ and the maximum temperature $(T)$ histories under the pressure of $P_{s}=200 \mathrm{MPa}, 300 \mathrm{MPa}$, and $500 \mathrm{MPa}$ are shown in Figures 5 and 6 . In the initial time the movement of the pore radius is the same as that of the Whitworth model. For example, the pore radius is $9 \mu \mathrm{m}$ at $t=10 \mu \mathrm{s}$ for both models when $P_{s}=500 \mathrm{MPa}$.

In general, the cracks in the explosive can expand gradually under continuing external forces. Therefore the damage level $(D)$ increases gradually and approaches 0.9 at last, as shown in Figure 7. During this process the balance of the movement of the pore may be broken because the strength of the explosive declines. Then, the pore radius decreases rapidly and the maximum temperature reaches $1000 \mathrm{~K}$ at the states of $P_{s}=500 \mathrm{MPa}, t=1500 \mu \mathrm{s}: P_{s}=300 \mathrm{MPa}, t=$ $2000 \mu \mathrm{s}$ : and $P_{s}=200 \mathrm{MPa}, t=2500 \mu \mathrm{s}$. In these cases, "hot-spots" can probably be generated under $P_{s}=200 \mathrm{MPa}$ with the improved model because of the effect of damage of explosives. On the contrary, "hot-spots" cannot appear under $P_{s}=500 \mathrm{MPa}$ with the Whitworth model. Thus, in the improved model the weaker pressure with longer loading time can also generate "hot-spots." This may be much more 


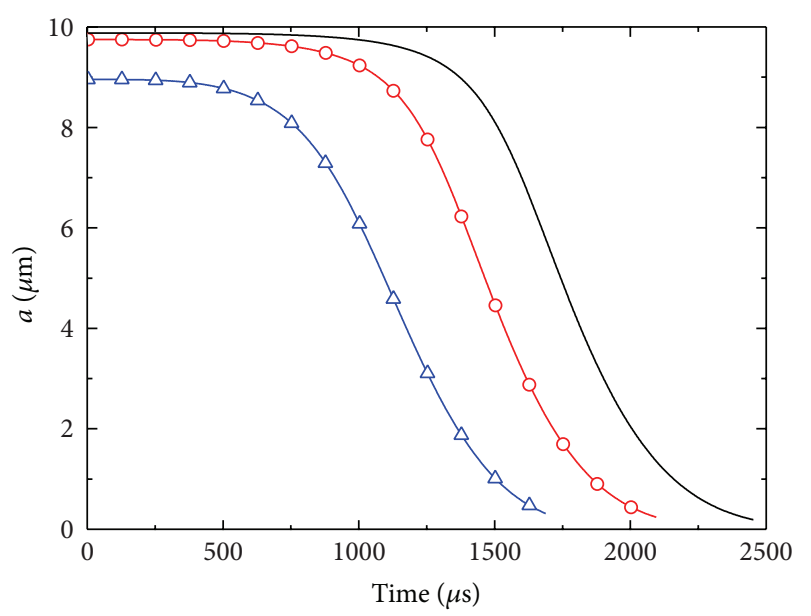

$$
\begin{aligned}
& -P_{s}=500 \mathrm{MPa} \\
& \multimap-P_{s}=300 \mathrm{MPa} \\
& -P_{s}=200 \mathrm{MPa}
\end{aligned}
$$

FIGURE 5: Time evolution of pore radius under lower pressures with the improved model.

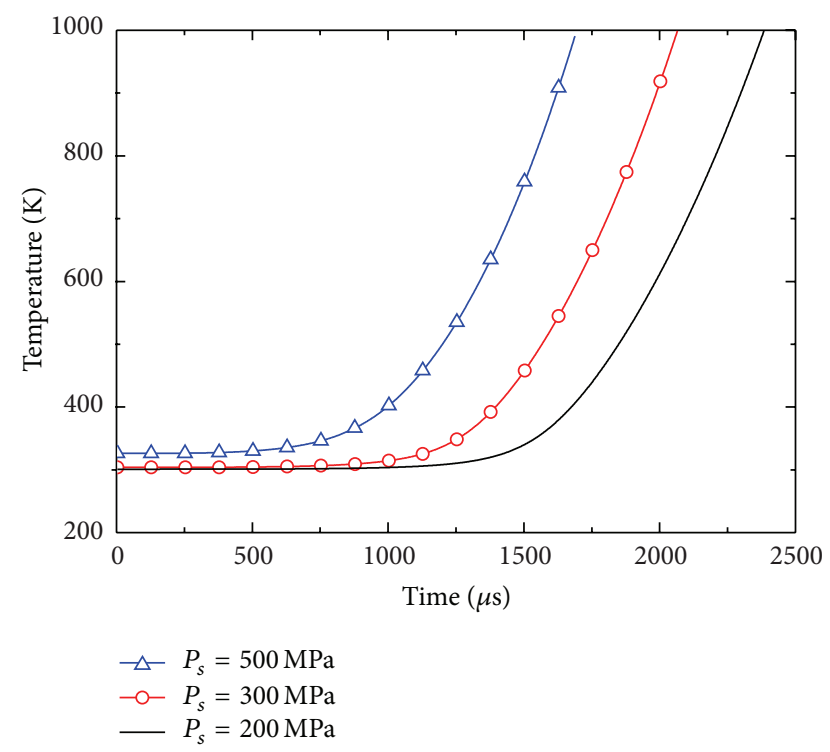

FIgURE 6: Time evolution of temperature at pore surface under lower pressures with the improved model.

approximate to the practice. However, "hot-spots" cannot occur if the external pressure drops to a very low level, such as $P_{s}=100 \mathrm{MPa}$, because the explosive is on the elastic state during the whole loading period. In this case, the stress intensity factor cannot exceed the fracture toughness and the average crack radius increases very slowly. According to the simulated results, one can see that the external loading with higher pressure can generate "hot-spots" within less time, whereas that with lower pressure needs more time to generate "hot-spots."

As shown in Figure 8, the external loading with four pulses (each pulse lasts for $500 \mu \mathrm{s}$ and the interval is $4500 \mu \mathrm{s}$ )

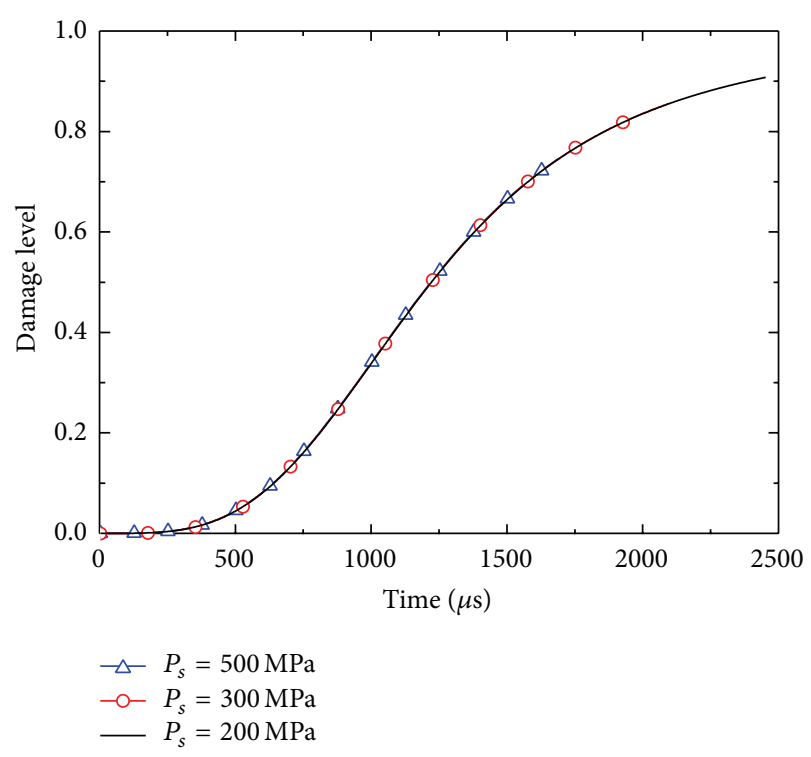

FIGURE 7: Time evolution of damage level under different pressures with the improved model.

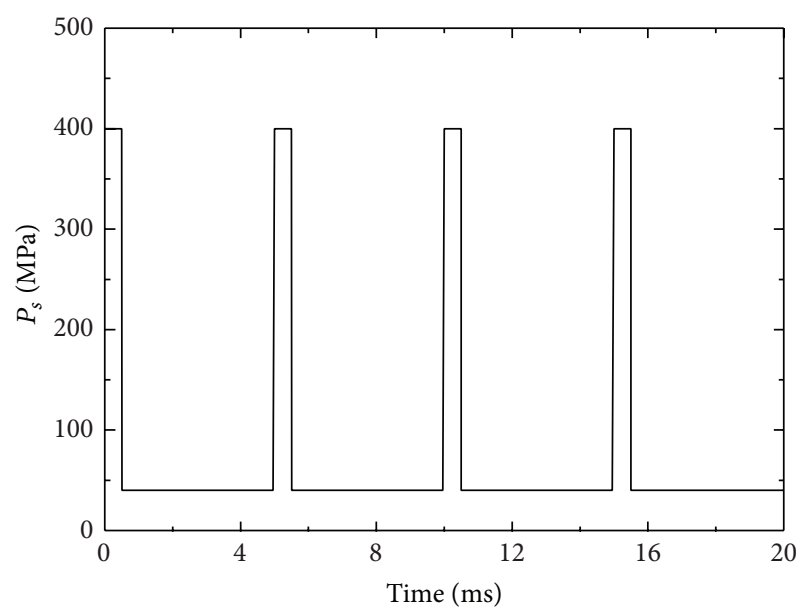

FIGURE 8: The loading history with four repeated pulses.

is used to predict "hot-spots." Using the improved model, the damage level $(D)$, the pore radius $(a)$, and the maximum temperature $(T)$ histories are calculated, as shown in Figures 9-11, under three different pressures $\left(P_{s}=200,300\right.$, and $400 \mathrm{MPa}$ ). When the explosive was loaded by the first loading pulse, the pore radius declined slightly; the material was nearly undamaged and in the elastic-viscoplastic state. When the first external loading was removed, the inner crack stopped expanding and the pore radius maintained a certain position (such as $a=9.4 \mu \mathrm{m}$ when $P_{s}=400 \mathrm{MPa}$ ). When the second pulse was applied to the explosive the crack continued to expand and the decline of the pore radius was slightly larger than that under first pulse. As above, when the second pulse ended, the inner radius also maintained current position. Under the third pressure, the decline of pore radius was larger than the previous two states. When the fourth pulse was applied, the pore radius rapidly 
TABLE 2: Results of multi-impulse impact tests of pressed PBX charge [20].

\begin{tabular}{lccccc}
\hline Test type & $1 \#$ & $2 \#$ & $3 \#$ & $4 \#$ & $5 \#$ \\
\hline The first impact & Safe & Safe & Safe & Safe & Safe \\
The second impact & Deflagration & Safe & Deflagration & Safe & Safe \\
The third impact & - & Deflagration & - & Deflagration & Deflagration \\
\hline
\end{tabular}

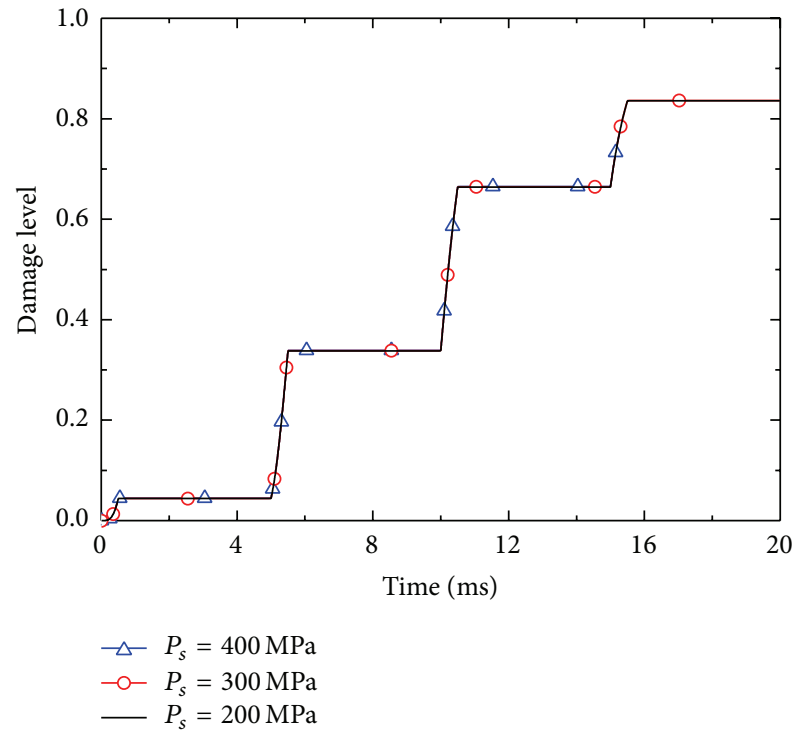

FIGURE 9: Time evolution of damage level when the explosive was loaded by four repeated pulses.

declined to around one-twentieth of the initial pore radius because the cumulated cracks had softened the explosive. Subsequently, the maximum temperature at the pore surface increased, which may motivate "hot-spots." Time evolution of the damage level $(D)$ and the pore radius $(a)$ is shown in Figures 9 and 10. The time evolution of the temperature at the pore surface is shown in Figure 11. As in the figures, one can see that the maximum temperature could rise to $620 \mathrm{~K}$ at $P_{s}=200 \mathrm{MPa}$ and rise to $920 \mathrm{~K}$ at $P_{s}=300 \mathrm{MPa}$. When $P_{s}=400 \mathrm{MPa}$, the maximum temperature exceeded $950 \mathrm{~K}$. In this case, the "hot-spots" could appear easily.

\section{Discussions}

To study the impact sensitivity of explosives, Gao et al. [20] carried out a medium-scale penetration test against multilayer targets, where the charge was in deflagration with the impact velocity of $579 \mathrm{~m} / \mathrm{s}$. Since the explosive charge was damaged during the first impact, its deflagration could happen more easily when it was loaded by the repeated impacts.

The experimental phenomenon can also be proven by the drop hammer tests. In the multi-impulse impact tests, five PBX specimens were tested under the same loading. Dimensions of the five specimens were all $\Phi 40 \mathrm{~mm} \times$ $40 \mathrm{~mm}$. The testing results are listed in Table 2 . Under the first external impact, all five specimens were safe and no

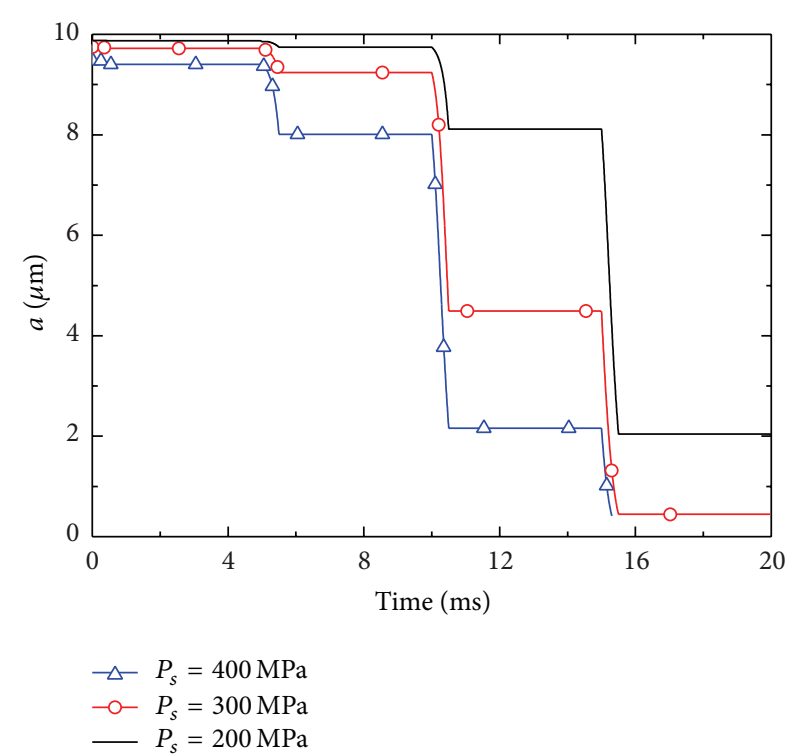

FIGURE 10: Time evolution of pore radius when the explosive was loaded by four repeated pulses.

deflagration occurred but there were visible cracks in each specimen. When the specimens are loaded by the second external impact deflagrations occurred in two specimens. Furthermore, the three other specimens are detonated when the third impact is applied to them.

According to the test results, repeated impacts can easily make the explosive generate "hot-spots." To obtain more details, the multilayer target test of penetrating warhead charge was simulated using LS_DYNA. In the simulation, the four-layer target was made of concrete, the thickness of the first layer was $300 \mathrm{~mm}$, and the others all were $200 \mathrm{~mm}$. The penetrating warhead weighed $450 \mathrm{~kg}$ and the proportion of length and diameter was 4.1. The impact velocity of the warhead was set to be $579 \mathrm{~m} / \mathrm{s}$, the same as the data of the experiments done by Gao et al. [20]. The penetration process of the simulation was shown in Figure 12. The pressure in the head charge can be extracted from the results of the simulation, as shown in Figure 13. Using the data, the maximum temperatures at pore surface can be calculated with the new model and Whitworth model and are shown in Figure 13. It can be seen that the maximum temperature (T) calculated with the new model rises to $850 \mathrm{~K}$ when the warhead is penetrating the second layer of the target. This may generate "hot-spots" in the explosive. However, the maximum temperature obtained from the Whitworth model only rises to the plateau of $310 \mathrm{~K}$ with the pore radius (a) remaining constantly $0.95 \mu \mathrm{m}$. Consequently, the improved 


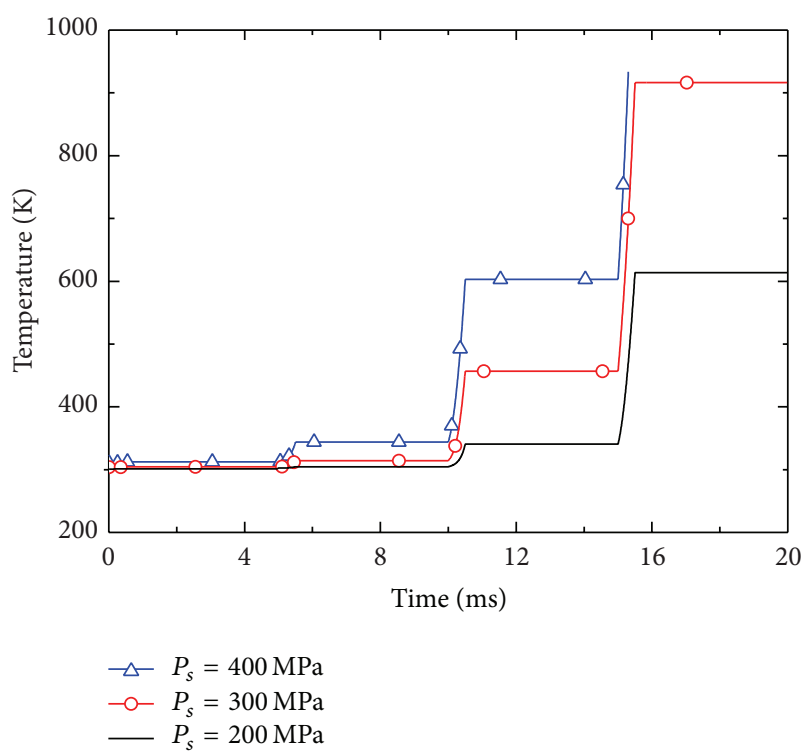

Figure 11: Temperature at pore surface when the explosive was loaded by four repeated pulses.
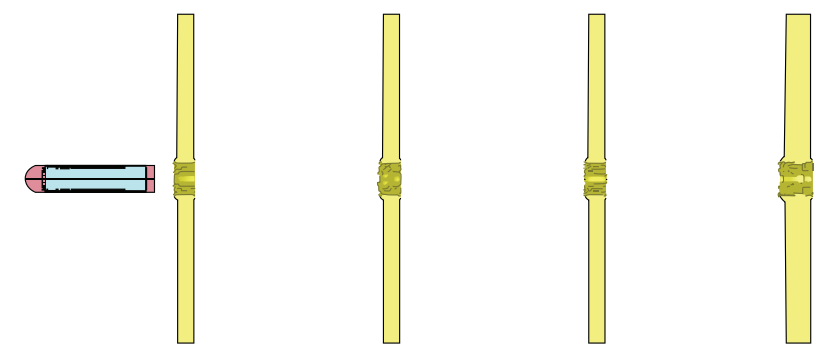

FIGURE 12: Simulation results of warhead penetrating into four-layer target $(v=579 \mathrm{~m} / \mathrm{s})$.

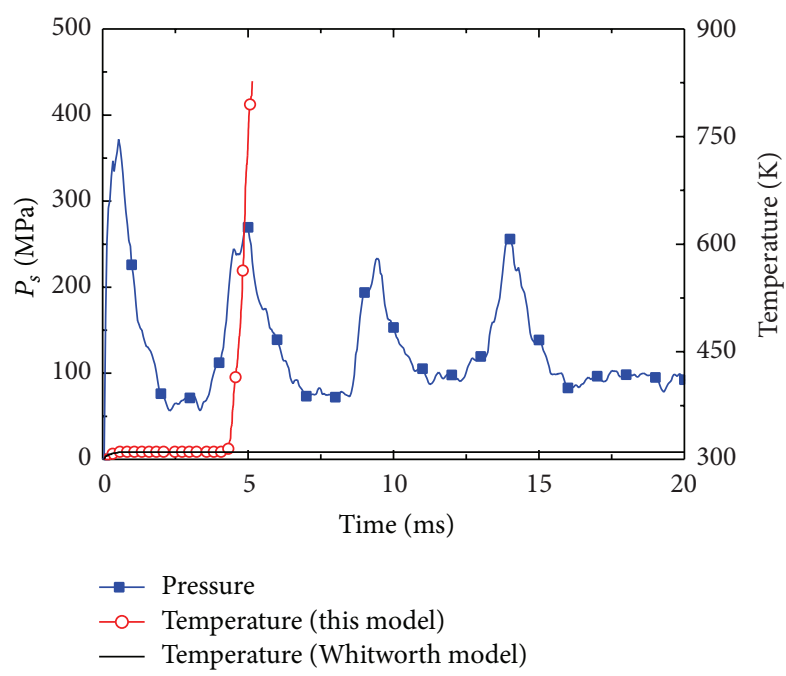

FIGURE 13: The pressure histories of the head charge in the simulation and temperature histories calculated with the two models. The "hot-spots" are generated during penetration in the second layer of the target with this model while the temperature remains $310 \mathrm{~K}$ with the original model. model is capable of explaining the XDT phenomenon when explosives are impacted repeatedly.

In the experiments carried out by Gao et al., the warhead exploded while it was penetrating the fourth layer of the concrete target. This is different from our simulated result. The difference may be interpreted in two aspects. One is that the material parameters used in the simulation cannot reflect the real properties of explosives; the other is that the buffering layers sandwiched between the explosive charge and the shell are ignored in the simulation. However, our model is capable of explicating the XDT phenomenon to some extent when the explosive is impacted repeatedly.

\section{Conclusions}

In the paper, an improved model based on the onedimensional elastic-viscoplastic collapse model was developed, in which brittle damage of explosives was considered. In the model, the collapse of the pore due to the explosives strength decline was thought of as the main cause to the "hot-spots." Using the improved model, the pore collapse and temperature of the explosive under different external loading were analyzed. The results showed that (1) the "hot-spots" could appear under moderate and weak loading pulse, which lasted for a longer time or was repeated. (2) To stimulate "hot-spots," the external loading with higher pressure needs less time, whereas the weaker loading needs more time. (3) Expansion of internal defects and damage accumulation under repeated external pressure are the main factors influencing the formation of "hot-spots." (4) A comparison of simulation results and experimental results in literature was made to be validated with the new model. Though there is a little difference between the modeling predictions and experimental results, the model indeed makes preliminary explanation for the XDT phenomenon. Furthermore, in the future work, the model may be improved regarding more damage details, if possible, and it can be introduced to some related kinetics programs to evaluate the safety of explosives in variety of warheads.

\section{Conflict of Interests}

The authors declare that there is no conflict of interests regarding the publication of this paper.

\section{Acknowledgments}

This work was supported by the Open Foundation of State Key Laboratory of Explosion Science and Technology (Beijing Institute of Technology, Grant no. KFJJ11-9Y) and the Natural Science Foundation of China (NSFC) through Grant nos. 11202232,11172328 , and 11132012.

\section{References}

[1] E. M. Olsen, J. T. Rosenberg, J. D. Kawamoto et al., "XDT investigation by computational simulations of mechanical response using a new viscous internal damage model," in Proceedings 
of the 11th International Detonation Symposium, pp. 170-178, Snowmass Village, Colo, USA, 1998.

[2] L. G. Green, E. James, and E. L. Lee, "Energetic response of propellant to high velocity impact," in Proceedings of the 8th International Detonation Symposium, pp. 284-293, 1985.

[3] E. R. Matheson and J. T. Rosenberg, "The role of damage mode in delayed detonation of composite energetic materials," in Proceedings of the 12th International Detonation Symposium, pp. 230-242, 2002.

[4] J. Massoni, R. Saurel, G. Baudin, and G. Demol, "A mechanistic model for shock initiation of solid explosives," Physics of Fluids, vol. 11, no. 3, pp. 710-736, 1999.

[5] R. B. Frey, "The initiation of explosive charges by rapid shear," in Proceedings of the 7th International Detonation Symposium, pp. 36-42, 1981.

[6] M. E. Kipp, "Modeling granular explosives detonation with shear band concepts," in Proceedings of the 8th International Detonation Symposium, pp. 35-41, 1985.

[7] J. K. Dienes, "Frictional hot-spots and statistical crack mechanics," LA-UR-83-3278, 1983.

[8] P. M. Howe, G. J. Gibbons, and P. E. Webber, "An experimental investigation of the role of shear in initiation of detonation by impact," in Proceedings of the 8th International Detonation Symposium, pp. 294-306, 1985.

[9] K. Kim, "Development of a model of reactive rates in shocked composite explosives," in Proceedings of the 9th International Detonation Symposium, pp. 593-603, 1989.

[10] N. Whitworth, Mathematical and numerical modelling of shock initiation in heterogeneous solid explosives [Ph.D. thesis], Cranfield University, 2008.

[11] N. J. Whitworth, "Development of a simple model of "hotspot" initiation in heterogeneous solid explosives," in Shock Compression of Condensed Matter-2001, Pts 1 and 2, M. D. Furnish, N. N. Thadhani, and Y. Horie, Eds., pp. 991-994, American Institute of Physics, Melville, NY, USA, 2002.

[12] Z. Y. Zhang, S. Huan, F. Y. Lu et al., "Viscoplastic collapse mechanism of hot-spot formation in porous TNT explosives," Energetic Materials, vol. 2, no. 2, pp. 36-42, 1994.

[13] L.-J. Wen, Z.-P. Duan, Z.-Y. Zhang, Z.-C. Ou, and F.-L. Huang, "An elastic/viscoplastic pore collapse model of double-layered hollow sphere for hot-spot ignition in shocked explosives," Chinese Journal of High Pressure Physics, vol. 25, no. 6, pp. 493500, 2011.

[14] M. M. Carroll and A. C. Holt, "Static and dynamic porecollapse relations for ductile porous materials," Journal of Applied Physics, vol. 43, no. 4, pp. 1626-1638, 1972.

[15] J. Li, F. Lu, J. Qin et al., "Effects of temperature and strain rate on the dynamic responses of three polymer-bonded explosives," Journal of Strain Analysis for Engineering Design, vol. 47, no. 2, pp. 104-112, 2012.

[16] R. Chen, F. Dai, J. Qin, and F. Lu, "Flattened Brazilian disc method for determining the dynamic tensile stress-strain curve of low strength brittle solids," Experimental Mechanics, vol. 53, no. 7, pp. 1153-1159, 2013.

[17] P. J. Rae, S. J. P. Palmer, H. T. Goldrein, J. E. Field, and A. L. Lewis, "Quasi-static studies of the deformation and failure of PBX 9501," Proceedings of the Royal Society A: Mathematical, Physical and Engineering Sciences, vol. 458, no. 2025, pp. 22272242, 2002.

[18] P. W. Chen and F. L. Huang, The Theory and Application of Energetic Material, Beijing Institute of Technology Press, Beijing, China, 2006.
[19] Y.-Q. Wu and F.-L. Huang, "A microscopic model for predicting hot-spot ignition of granular energetic crystals in response to drop-weight impacts," Mechanics of Materials, vol. 43, no. 12, pp. 835-852, 2011.

[20] J. X. Gao, W. G. Zhao, and T. Zhen, "Study on the antioverloading technique for penetrating warhead charge," Initiators and Pyrotechnic, vol. 5, no. 4, pp. 4-7, 2008.

[21] C. B. Skidmore, D. S. Phillips, and P. M. Howe, "The evolution of microstructural changes in pressed HMX explosives," in Proceedings of the 11th International Detonation Symposium, pp. 556-564, 1998.

[22] J. G. Bennett, K. S. Haberman, J. N. Johnson, B. W. Asay, and B. F. Henson, "A constitutive model for the non-shock ignition and mechanical response of high explosives," Journal of the Mechanics and Physics of Solids, vol. 46, no. 12, pp. 2303-2322, 1998.

[23] P. Rangaswamy, D. G. Thompson, C. Liu, and M. W. Lewis, "Modeling the mechanical response of PBX-9501," in Proceedings of the 14th International Detonation Symposium, 2010, Paper no. 18277.

[24] J. K. Dienes, Q. H. Zuo, and J. D. Kershner, "Impact initiation of explosives and propellants via statistical crack mechanics," Journal of the Mechanics and Physics of Solids, vol. 54, no. 6, pp. 1237-1275, 2006.

[25] J. K. Dienes, Statistical Crack Mechanics, Los Alamos National Laboratory, Los Alamos, NM, USA, 1983.

[26] J. K. Dienes, "A unified theory of flow, hot spot, and fragmentation with an application to explosive sensitivity," in HighPressure Shock Compression of Solids II, D. Lee, Ed., pp. 366-398, 1996. 

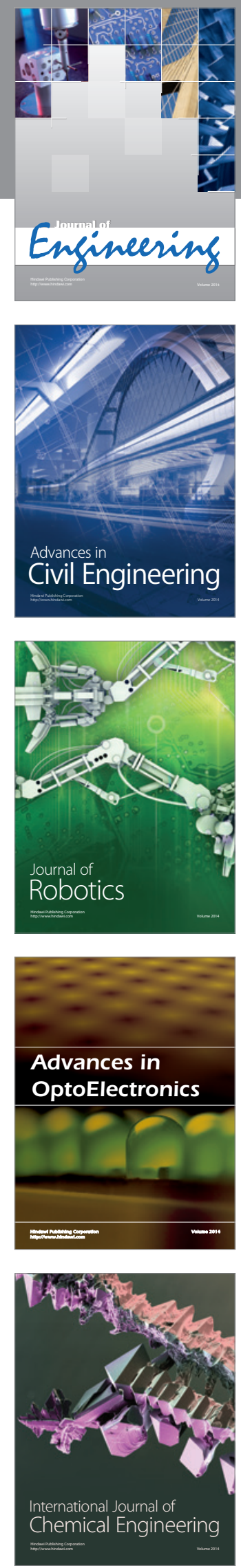

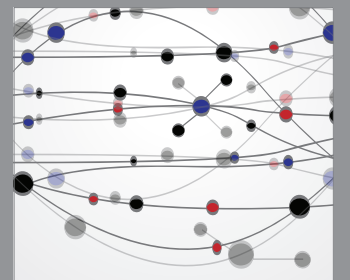

The Scientific World Journal
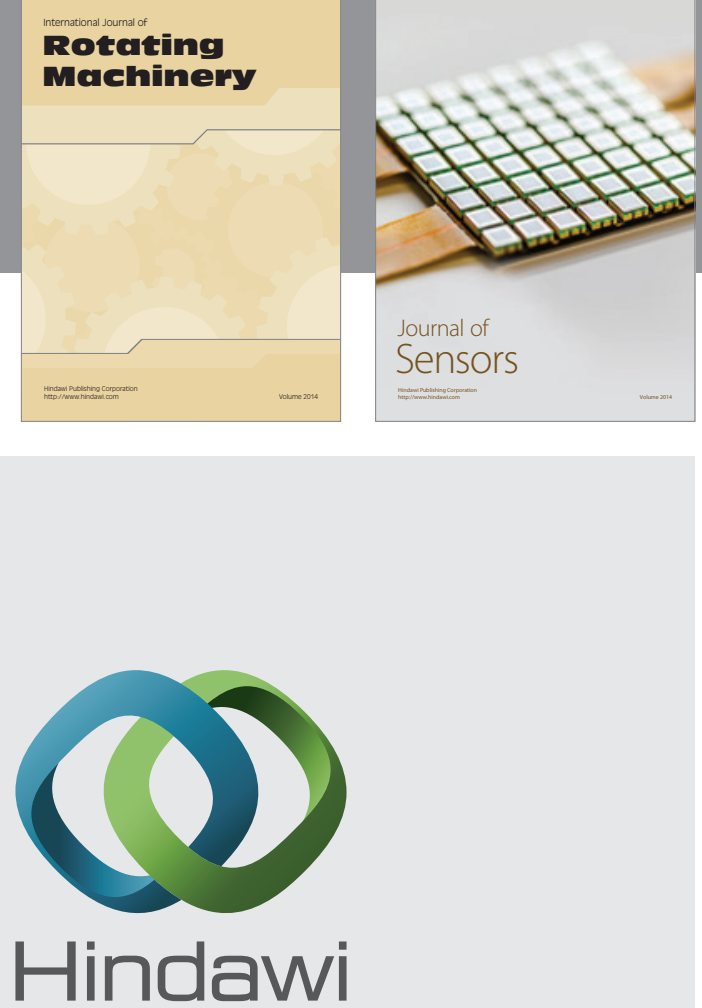

Submit your manuscripts at http://www.hindawi.com
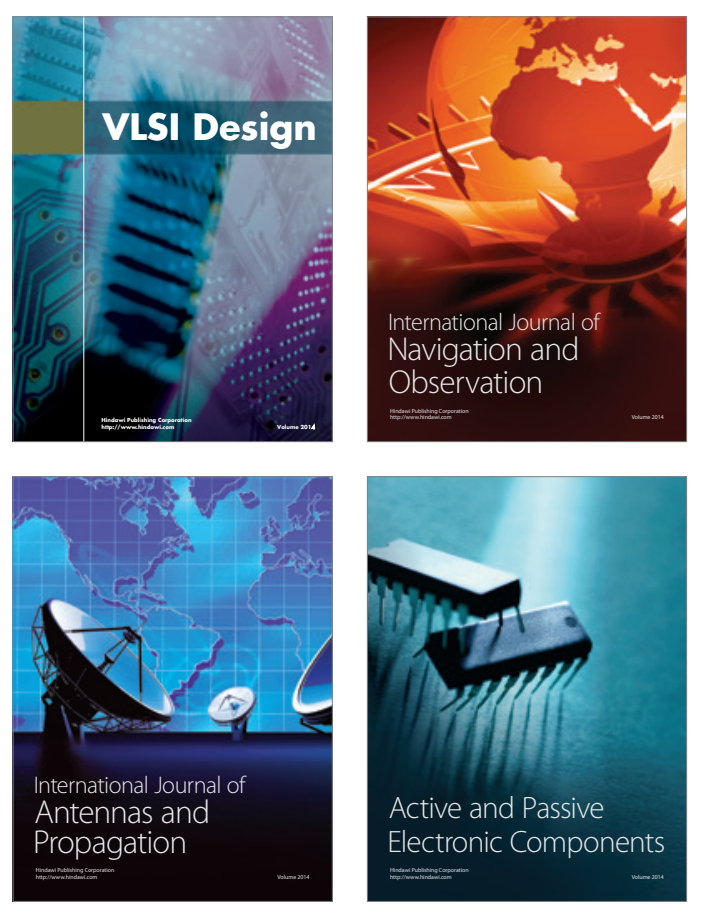
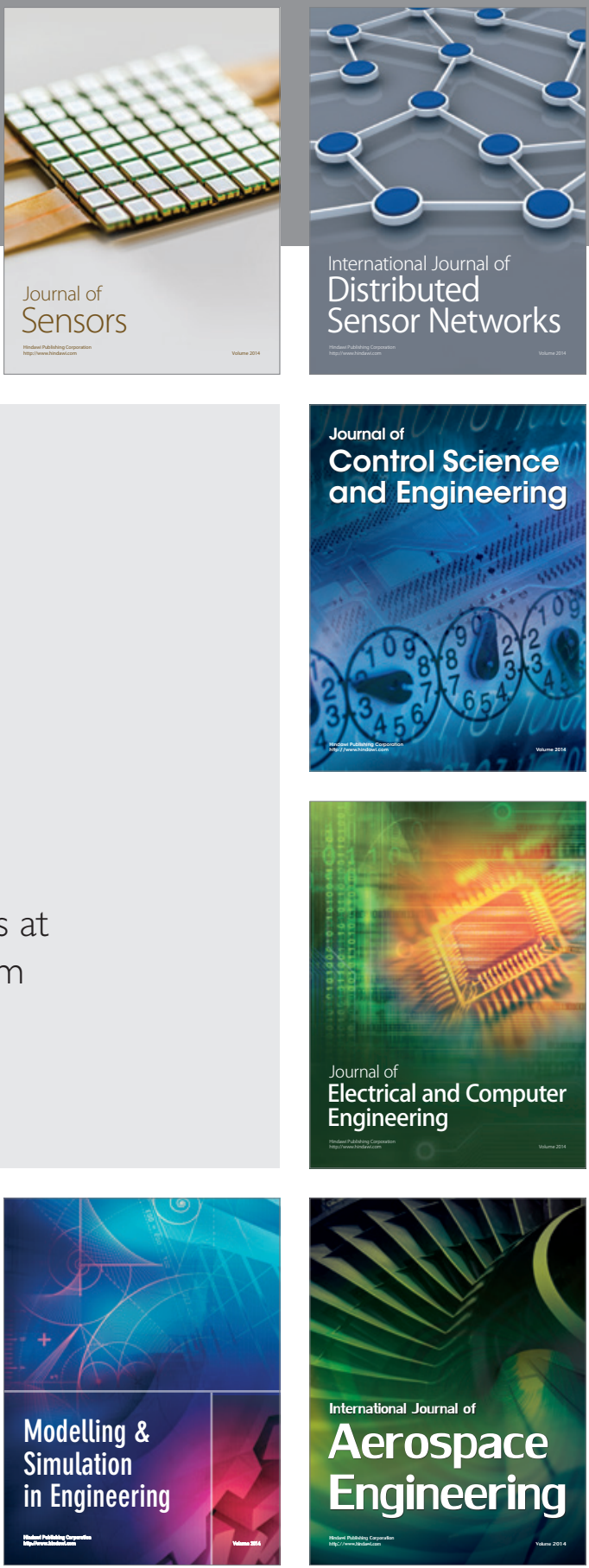

Journal of

Control Science

and Engineering
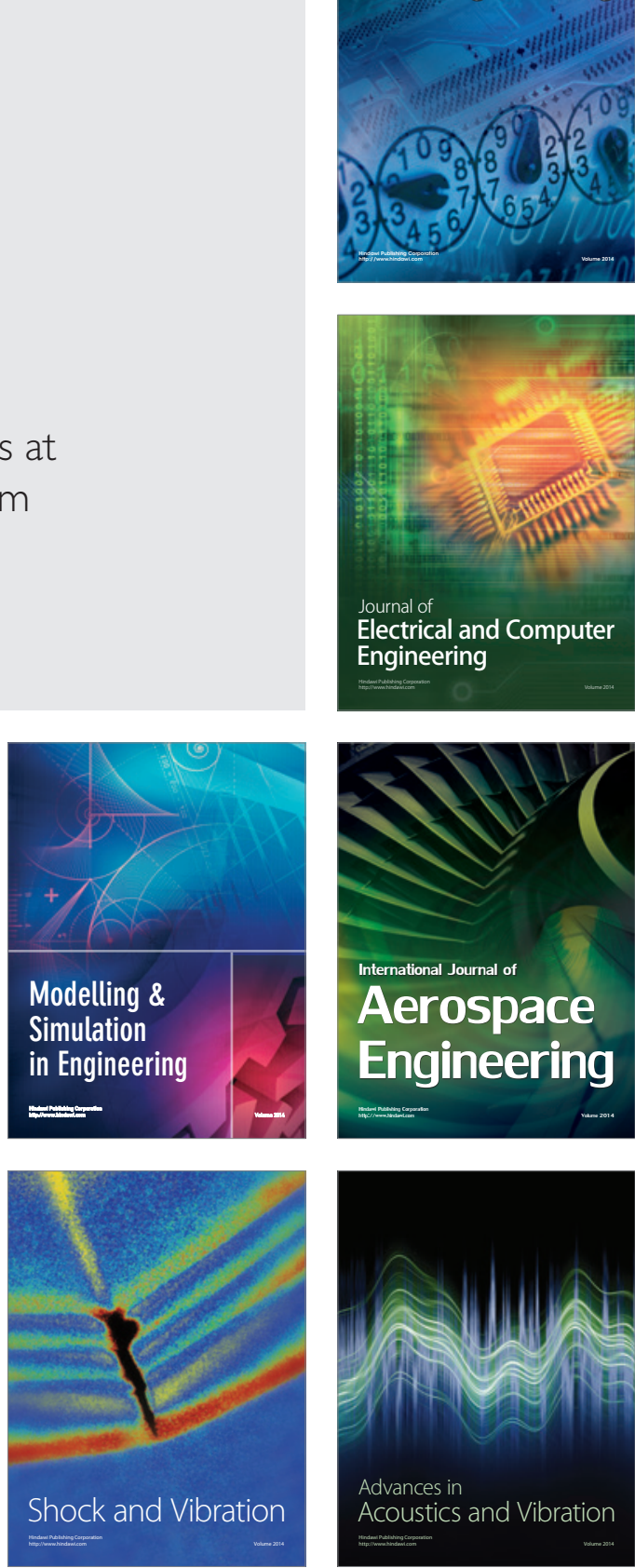